\title{
Mini-symposium
}

\section{Plaque stabilisation in coronary artery disease: introduction}

$\mathrm{P}$ laque vulnerability, thin cap fibroatheroma, plaque erosion, plaque rupture, plaque sealing, plaque passivation, plaque stabilisation-there appears to be no end to the neologisms pertaining to what makes atherosclerosis so treacherous. After all, arteries are everywhere. A tiny accident in a proximal coronary artery (or cerebral artery for that matter) may put an end to the perfect harmonious functioning of billions of healthy cells making up the human body. And it does so time and time again, so often that this is the most common cause for the end of human life. No wonder that a feverish global quest is on to know more about this enemy, anticipate its strikes, keep it from planting mines, and, perhaps, extinguish it for good. There is still a long way to go but we are on the road. Already have we learned that not all plaques pose a threat. A non-obstructive plaque with a thick fibrous cap rich in smooth muscle cells and poor in inflammatory cells and activated or apoptotic macrophages may be a man's best friend. It covers up a blemish in the arterial wall, namely a deposit of cholesterol (formerly alluded to as fatty streak), with aggressive metalloproteinases ready to erode the surface to get to the lumen. Such a plaque will remain incarcerated for ever-it is sealed. On the other hand, the active (vulnerable) plaque with activated macrophages, few muscle cells at the surface forming a thin and brittle cover, and many inflammatory cells and destructive enzymes near the surface represents a deadly menace.

The first contribution from the group which coined the term "thin cap fibroatheroma" succinctly delineates the problem from the histological point of view. It also hints at some biochemical mechanisms, none of which are carved in stone and many of which provide a handle to curb the problem.

The second contribution describes a logical approach, hailed at first but struck out for technical complexity, only to be revived very recently with drug eluting stents. Chemical stabilisation with local delivery provides the highest concentration of the agent where it is warranted without systemic side effects. However, it is difficult to get the compounds there, to make sure they stay there for the time needed, and last but not least to keep the blood flowing while the drugs are applied. The drug eluting stent miraculously solves all these problems. Targeted at reducing restenosis, it can clearly also contribute to plaque stabilisation in terms of preventing subsequent rupture and thrombotic occlusion.

The third contribution elaborates on the local approach by pointing out that splitting the plaque on purpose during coronary angioplasty fairly reliably turns a perhaps malignant species of a plaque into a benign (sealed) one.

It has been debated iteratively whether an expensive approach to a single or may be a few plaques by angioplasty is defendable in light of the multitude of coronary plaques to be suspected once the disease has started. First, the number of dangerous plaques in a coronary tree of a patient diagnosed with coronary artery disease normally can be counted on one hand. ${ }^{1}$ Second, only those plaques in a strategically important proximal location are of interest. Third, many plaques need to be treated anyway to re-establish normal flow and alleviate anginal symptoms. Finally, does it not make sense to apprehend and put away for good a single terrorist, even though you cannot be sure that this one would have caused evil in the future and you are sure that more terrorists are out there?

Correspondence to: Professor Bernhard Meier, Swiss Cardiovascular Center Bern, University Hospital, CH-3010 Bern, Switzerland; bernhard.meier@insel.ch

\section{REFERENCE}

1 Rioufol G, Finet G, Ginon I, et al. Multiple atherosclerotic plaque rupture in acute coronary syndrome. A three-vessel intravascular ultrasound study. Circulation 2002;106:804-8. 